\title{
LA INFLUENCIA DEL EMPIRIOCRITICISMO DE E. MACH EN LA FUNDACIÓN DE LA PSICOLOGÍA
}

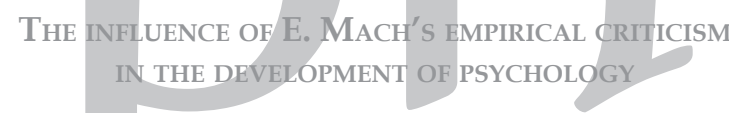

\author{
Flor Emilce Cely Á.*
}

Universidad El Bosque, Colombia. florcely@gmail.com

RECIBIDO EL 13 DE FEBRERO DE 2019, APROBADO EL 30 DE JULIO DE 2019

\section{Resumen Abstract}

El propósito de este artículo es presentar algunos elementos que permiten rastrear la influencia de Mach en el nacimiento de tres enfoques psicológicos: el conductismo skinneriano, el psicoanálisis freudiano y la psicología de la Gestalt. Se examina, en primer lugar, la particular interpretación que Skinner y Freud hicieron de algunos postulados de la filosofía de la ciencia de Mach. En la segunda parte se analiza la influencia de la obra de Mach sobre la psicología de la Gestalt y la fenomenología husserliana. Finalmente, se resalta la importancia que habría tenido para la naciente ciencia de la psicología haber realizado una interpretación correcta del plan de estudio de las sensaciones desde un punto de vista empírico y experiencial, en la convergencia entre la psicofisiología de Mach y el estudio de la experiencia consciente en fenomenología.

\section{Palabras clave}

Mach; positivismo; Skinner; Freud; Gestalt; fenomenología.
The purpose of this article is to present some elements that permit tracing the influence of Mach in the birth of three psychological approaches: Skinnerian behaviorism, Freudian psychoanalysis and Gestalt psychology. In the first place, the particular interpretation that Skinner and Freud made of some postulates of Mach's philosophy of science are examined. The second part analyzes the influence of Mach's work on the Gestalt psychology and the Husserlian phenomenology. Finally, the importance of having made a correct interpretation of the study plan of sensations, from an empirical and experiential point of view in the convergence between Mach's Psychophysiology and the study of conscious experience in phenomenology, that it would have had for the emerging science of psychology, is emphasized.

\section{KEY WORDS}

Mach, Positivism, Skinner, Freud, Gestalt, Phenomenology .

\footnotetext{
* iD orcid.org/0000-0003-3477-1922 Google Scholar
} 


\section{Introducción}

El propósito de este artículo es presentar algunos elementos que permiten rastrear la influencia de Mach en el nacimiento de tres enfoques psicológicos: el conductismo de Skinner, el psicoanálisis freudiano y la psicología de la Gestalt. En relación con Skinner y Freud, el énfasis estará puesto en mostrar, de manera crítica, la particular y desacertada interpretación que cada uno de ellos hizo de algunas de los postulados epistemológicos y científicos de Mach. En la segunda parte se analiza la influencia de la obra de Mach sobre la psicología de la Gestalt y la fenomenología husserliana. Finalmente, se resalta la importancia que habría tenido para la naciente ciencia de la psicología -tal como se estaba gestando en las primera décadas del siglo XX-, el haber realizado una interpretación correcta del programa de estudio de las sensaciones desde un punto de vista empírico y experiencial, tal como se hubiese articulado desde la psicofisiología de Mach y el estudio de la experiencia consciente en fenomenología, y no desde la sesgada interpretación que realizaron los padres del conductismo y del psicoanálisis.

\section{Principios epistemológicos de la ciencia en Mach}

La persona y la obra de Ernst Mach como científico y filósofo de la ciencia constituyeron un centro de gravedad para los intelectuales y científicos de la primera mitad del siglo XX. Ejerció influencia en autores tan diversos como Freud, Skinner, James y los fundadores de la psicología de la Gestalt, así como en el movimiento del positivismo lógico y en científicos como Einstein y Planck.

Como físico es conocido por cuestionar el paradigma mecanicista y defender una renovación de las categorías físicas al criticar las nociones de espacio, tiempo y movimiento absolutos de Newton. El cuestionamiento de estos conceptos de la mecánica newtoniana le valió la admiración de Einstein quien consideró el trabajo de Mach como un precursor filosófico de la teoría de la relatividad. Se cuenta a Mach entonces entre los grandes físicos que dieron origen no solo a nuevos desarrollos en física, sino a nuevas concepciones científicas acerca de la realidad y, en últimas, a toda una nueva concepción de la naturaleza. Y esto estuvieron dispuestos a reconocerlo grandes físicos de la época como Einstein y Planck. Para ellos, La ciencia de la mecánica fue una obra excepcional que abrió el camino para que sus propios trabajos revolucionarios fueran recibidos de buena forma por la comunidad de 
físicos (a pesar de la distancia que después los separó por cuenta del tema de la teoría atómica).

Mach cuestionó el paradigma mecanicista newtoniano con una actitud al mismo tiempo de científico y de epistemólogo. Rehusó siempre considerarse filósofo, y prefería describir sus reflexiones desde este el último punto de vista más bien como de sabio. Para ello se sirvió de las investigaciones en biología y psicología experimental (Fechner). De hecho, su interés estuvo orientado a integrar investigaciones de orden físico y psicológico, por lo que se inclinó a encontrar una vía de continuidad entre la física y la psicología: "De acuerdo con Mach, la ciencia no es más que la comparación y ordenamiento de los contenidos de consciencia dados factualmente, mediante ciertos métodos y puntos de vista gradual y tentativamente probados. Por lo tanto, la física y la psicología deben distinguirse entre sí no por los objetos que estudian, sino solo por la forma de ordenarlos y relacionarlos" (Einstein, Ernst Mach 155).

De ahí su concepción fenomenista respecto al conocimiento. Todo conocimiento se origina en las sensaciones, su interés se centró entonces en el análisis de estas y su relación con los estímulos físicos que las desencadenan. Tan es así que escribe mucho más sobre la psicología del espacio que sobre la física del espacio. Con ello, no hacía más que sentar su posición monista, no solo a nivel ontológico, sino epistemológico, enlazando con ello la línea de estudio de la psicología y la física: “...uno reconocerá los elementos comunes más simples de ambos campos, que en el dominio psicológico llamamos sensaciones y que en las ciencias naturales se conocen como cualidades físicas, pero que en realidad son idénticos y solo son diferentes en las diferentes formas en que se consideran las relaciones" (Mach, Draft 116).

Su sistema es conocido como empiriocriticismo o crítica de la experiencia puesto que se propuso estudiar la experiencia humana, prescindiendo de las discusiones asociadas con los conceptos -para él metafísicosde sustancialidad, causalidad y necesidad; de ahí que se lo presente como un representante del positivismo. No obstante, no se lo puede identificar como un heredero del positivismo de Comte ya que este despreciaba la psicología, mientras para Mach ésta tenía igual importancia que la física. Y tampoco como un miembro activo del movimiento del positivismo lógico pues, aunque Mach haya sido una real influencia para la conformación de este movimiento y coincidiera con sus miembros en 
Flor Emilce Cely Á.

rechazar la metafísica, la actitud hacia ésta fue muy distinta: para los miembros del círculo de Viena cualquier enunciado de este tipo quedaba por fuera de la ciencia, mientras que para Mach...

Era precisamente lo que parecía metafísico o sin sentido, como lo no relacionado aún, lo que invitaba a la investigación científica con el fin de hacerlo significativo encontrando relaciones con lo que es sensorial o empírico. Lo metafísico o lo sin sentido como lo aún no experimentado, o aún no entendido, o incluso como lo que parecía no apto para las dos cosas, era para Mach una oportunidad o un desafío para la ciencia, pero para los positivistas estaba fuera de la ciencia. (Blackmore XVII)

En consecuencia, hay que tener muy en cuenta las particularidades de su estilo propio de positivismo pues, de hecho, estuvo más cerca del pensamiento de autores como James y Husserl que de filósofos como Comte o Schlick. Además, su particular 'filosofía de la ciencia' se basó enteramente en su propia práctica científica: se construyó a partir de lo que la experiencia le enseñó como científico. Por ello fue muy crítico de aquellos que pretendían establecer de antemano los requisitos o criterios para que algo pudiera ser considerado propiamente 'científico' (tales como los que defendían criterios a priori de racionalidad científica, y que incluyen por igual a positivistas lógicos y a sus detractores).

Voy a presentar en seguida algunos postulados epistemológicos centrales en Mach, para luego pasar a mostrar la influencia y peculiar interpretación de los mismos en Skinner y Freud.

En primer lugar, Mach consideraba el progreso del conocimiento científico en estrecha relación con su evolución histórica. Con ello pretendía mostrar que los elementos metafísicos no son esenciales en la ciencia. Por ejemplo, consideraba que el concepto de fuerza, central en la mecánica clásica, y que se extendió y refinó durante siglos, surgió de la experiencia en el trabajo artesano diario, es decir, para Mach, tenía un claro origen experiencial. "La actividad biológica más simple y natural es suficiente para establecer una base para el desarrollo de la física primitiva. Para el hombre primitivo bajo condiciones primitivas, la reacción de los objetos a su alcance sobre su propio cuerpo es más importante; y esta es su experiencia más frecuente" (Mach, Sensory Elements 121) 
De esta concepción evolucionista de la ciencia se desprende en Mach su principio de economía de la ciencia. Este principio se entiende como uno de simplicidad lógica. Para Mach, toda actividad humana, incluida por supuesto la ciencia, es un medio para la supervivencia.

Expresado de la manera más breve, la tarea del conocimiento científico parece ser: adaptar pensamientos a hechos y pensamientos entre sí. Todo proceso biológico favorable es uno de auto conservación, y como tal es al mismo tiempo un ajuste y más económico que un proceso que es desventajoso para el individuo. Todo conocimiento beneficioso consiste en casos especiales o partes de procesos biológicamente útiles (Mach, Leading Thoughts 133-134).

Esto es lo que hoy se conoce como la parsimonia intelectual que es continua con la economía de las necesidades biológicas.

En segundo lugar, tenemos el rechazo de Mach a la noción de causa, derivada del mecanicismo como algo que es eficiente en producir sus efectos. La rechaza dado que se trata de una noción metafísica que introduce elementos especulativos no observables y no comprobables. Y propone más bien entender los fenómenos como correlacionados, y la labor científica como encargada de describir las relaciones de interdependencia entre ellos.

Cuando las ciencias están muy desarrolladas emplean más raramente los conceptos de causa y efecto. La razón está en que tales conceptos son provisorios, incompletos e imprecisos. Desde que se llega a caracterizar los hechos por magnitudes mensurables (...) la noción de función permite representar mucho mejor las relaciones de los elementos entre sí (Mach, Conocimiento y error 219).

La labor de la ciencia consistiría entonces en describir estas relaciones funcionales. Así, la labor científica no se distrae ya en la búsqueda de causas eficientes, animada como estuvo por la promesa filosófica del hallazgo de unas conexiones universales y necesarias que, para Mach, constituían más bien temas metafísicos.

Y finalmente está el polémico planteamiento de Mach en relación con el papel que cumplen las hipótesis y teorías en la investigación científica. Para nuestro autor, una ciencia descriptiva puede proceder sin hipótesis explicativas o teorías. De hecho, Mach concebía a las teorías simplemente 
como resumen de hechos observables. Fue justamente esta obstinación la que llevó a Einstein a hacerle fuerte críticas. El físico alemán había reconocido en el trabajo científico de Mach una primera y gran influencia, pero no dejó de reclamarle después el hecho de que "...no situó bajo la luz correcta la naturaleza esencialmente constructiva y especulativa del pensamiento y especialmente del pensamiento científico; como consecuencia de lo cual condenó la teoría precisamente en aquellos puntos en los que su carácter constructivo-especulativo inesperadamente sale a la luz, como por ejemplo en la teoría cinética atómica" (Einstein, "Autobiographical Notes", citado en Cappelletti 218).

Este se puede considerar como uno de los puntos más desafortunados de la filosofía de la ciencia de Mach, uno que justamente el posterior avance de la física se encargaría de mostrar como errado, pues se trató nada menos que de un rechazo a una vía de avance científico que se basó en postular entidades inobservables (como, por ejemplo, los átomos) basado en el supuesto fuerte de que aquello que no puede ser observado debe ser desechado del campo de estudio científico.

Hay que entender en todo caso que el rechazo de Mach de la teoría atómica tuvo que ver con su actitud radicalmente crítica ante cualquier dogma y esta es una actitud nada despreciable. En la polémica con Planck quedan claros los motivos de recelo y desconfianza de Mach respecto a la nueva y exaltada teoría atómica ya que, en principio, se trataba de que aceptara una entidad teórica que a él le parecía dudosa. Esto le costó la crítica acérrima de Planck quien, incluso, lo llamo un falso profeta. De manera tal que se entiende la reacción de Mach al afirmar que prefería quedarse por fuera de esa "comunidad de creyentes" que lo excluía de esta manera por no adherirse a un dogma, según él, aceptado tan acríticamente (Cf. Mach, Leading Thoughts 138).

\section{Skinner}

Mach hizo énfasis en la evolución histórica de las ciencias y en tal tarea entraron en consideración de manera importante los datos de la historia de los conceptos científicos que pueden interpretarse en un cierto sentido como refiriéndose al carácter contingente de leyes y conceptos. Esta última idea fue acogida por Skinner al defender que las proposiciones de la ciencia son todas contingentes porque están basadas en la experiencia. Pero, como veremos más adelante, la concepción de "experiencia" de Skinner es una bastante simplificada y alejada de la rica 
y compleja noción de experiencia en Mach. El fundador del conductismo veía a la actividad científica como un tipo especial de conducta que, al igual que toda conducta, está gobernada por las contingencias de refuerzo y supervivencia. Tal vez esto explica las prácticas científicas bastante particulares que llevó a cabo, en las que los "accidentes fortuitos" adquirieron una relevancia particular. Por ejemplo, la idea de "curvas de extinción constante" surgió en su investigación por accidente, debido a la falla de una pieza del equipo. O los "programas de reforzamiento intermitente" fueron descubiertos cuando Skinner se vio obligado a suspender el refuerzo de cada respuesta del animal debido a la carestía de bolitas de comida (Cf. Smith 300).

Además de esta particular forma de improvisación, lo que podemos apreciar es que Skinner olvidó los orígenes fenomenistas del concepto de experiencia -que para Mach eran fundamentales- y entendió "experiencia" en un sentido básico de recepción de estímulos por parte de organismos pasivos que cambian su comportamiento en relación con las repeticiones de consecuencias positivas o negativas. Esto lo llevó a defender la idea de que la conducta de humanos y animales está gobernada por asociaciones adquiridas por la experiencia y mantenidas por su utilidad biológica.

Asimismo la particular lectura que hace Skinner de Mach lo lleva a simplificar el principio de la "economía de la ciencia" al enfatizar, por ejemplo, la inmediatez de la observación, la economía de la descripción y la comunicación ${ }^{1}$ y la eficacia de la investigación, en el sentido de no aspirar a la explicación, ni siquiera a la predicción, sino al control (Skinner Sobre el conductismo 8). Ofreció el paquete completo de una tecnología de la conducta que aportaba los medios para modificar y diseñar conductas a pedido del usuario a través de la manipulación de las variables independientes. ${ }^{2}$

Es conocida la idea de que Mach defendió más la labor científica como una labor descriptiva y no explicativa. Pero esta actitud tenía todo que ver con su trabajo como físico en el que se ocupaba más de describir

\footnotetext{
${ }^{1}$ Esto se puede apreciar claramente en la propuesta de eliminar los términos 'mentalistas' de las descripciones de la conducta humana, utilizando en su lugar una terminología técnica empobrecida que, como dijo Chomsky, al trasladarse del laboratorio pierde toda su significación y no aporta ninguna precisión (Chomsky 39 y 45).

${ }^{2}$ Cf. por ejemplo Tecnología de la enseñanza, en donde ofrece modificar la conducta de los estudiantes a partir de sus leyes de reforzamiento, dejando de lado el lenguaje mentalista de motivaciones, intenciones, etc. (para él arcaico).
} 
los fenómenos que manipulaba experimentalmente, rechazando un punto de partida especulativo. De ahí que defendiera la idea de que describir los fenómenos adecuadamente equivalía a explicarlos. Esto también fue recogido por Skinner en un primer momento, asociándolo con el reemplazo de la explicación causal mecanicista de los fenómenos por una descripción de sus relaciones funcionales. Ahora bien, Skinner pretendió hacer una analogía de la psicología con la física. Esta abandonó la idea de explicar el movimiento de los cuerpos de una manera animista y antropomórfica y, de igual manera, se suponía que la psicología, para ser reconocida como ciencia debía abandonar no solo el lenguaje de motivaciones e intenciones, sino la noción misma de causalidad (Skinner Beyond Freedom 13-14). Y la alternativa que propuso fue la explicación funcional que apela a causas funcionales de la conducta. En esto Skinner sigue la orientación de Mach en el sentido de reemplazar la noción de causalidad entendida como fuerza interna o agencia, por una relación funcional entre una variable dependiente, la conducta y una independiente, las variables ambientales.

Una "causa" pasa a ser un "cambio en una variable independiente" y un "efecto" un "cambio en una variable dependiente". La vieja "conexión de causa-y-efecto" pasa a ser una "relación funcional". Los nuevos términos no sugieren cómo una causa causa su efecto; ellos solamente afirman que eventos diferentes tienden a ocurrir juntos en un cierto orden (Skinner, Science and Human Behavior 23).

En contraste con la causalidad mecánica que requiere de causas iniciadoras, Skinner defendió entonces un tipo de "causalidad consecuencial" según el cual el comportamiento es una función de consecuencias ambientales pasadas, esto es, variables que seleccionan o descartan una conducta determinada (Skinner Sobre el conductismo 39). En este modelo, explicar la ocurrencia de un evento es relacionarlo con sus causas, pero en el sentido de citar la relación funcional que ya mencionamos. Así, el comportamiento es explicado describiendo dependencias funcionales dentro de los datos más que invocando propiedades o entidades inobservables.

Orientar la psicología hacia la búsqueda de las relaciones funcionales de la conducta con sus variables ambientales llevó a Skinner a un exagerado rechazo de los estados mentales, so pretexto de que estos no podían ser estudiados científicamente. Pero la revolución cognitiva llevada a cabo en la década de los 50 y la constante evolución de este 
enfoque y su colaboración interdisciplinaria con las neurociencias y la filosofía, han demostrado que tal rechazo no era necesario y que no es tampoco deseable. Podemos apreciar aquí que la mera importación de un principio metodológico de la ciencia física no le sirvió a la psicología en su proyecto de convertirse en una 'ciencia de la conducta', puesto que lo que se logró con ello fue, de un lado, poner en evidencia lo vano que resulta la imposición de principios aplicados con éxito en el campo de los fenómenos físicos al ámbito de un fenómeno cualitativamente diferente y complejo como es la conducta. Y, de otro, desfigurar el objeto de estudio de la psicología, reducirlo a un concepto simplificado de comportamiento en el que se ignora su estructura de funcionamiento como un todo (Merleau-Ponty Estructura del comportamiento 140-153).

Finalmente, examinemos cómo Skinner asimiló el postulado machiano del lugar e importancia de las hipótesis teóricas en la ciencia. Skinner rechazó las teorías planteadas en términos mentalistas o conceptuales pues las consideraba simplemente inútiles, así como también las teorías planteadas en términos fisiológicos, al ser, según él, irrelevantes para ciencia de la conducta ${ }^{3}$. No es seguro que Skinner haya hecho una correcta asimilación del postulado machiano y, aunque así fuera, es una lástima que haya acertado justamente en la interpretación de uno de sus principios epistemológicos más cuestionados y que la historia se encargó de desvirtuar. Tanto en la historia de la física moderna como en la de la psicología los puntos de vista teóricos, así como la aceptación de entidades teóricas, han enriquecido la investigación científica en varios momentos cardinales.

Más allá de esto, hay que ver que en Mach se unió el genio del físico con una visión universal del conocimiento: entendió que se puede avanzar en ciencia solo si al mismo tiempo se reflexiona sobre la manera en que ésta debe hacerse $e^{4}$. De ahí su vivo interés por la psicología fisiológica de la época, a la cual se dirigió con el fin de entender los elementos últimos

\footnotetext{
3 “...no podernos anticipar lo que hará una persona mirando directamente sus sentimientos o su sistema nervioso, ni podemos cambiar su comportamiento cambiando su mente o su cerebro. En cualquier caso, no parece que estemos peor si ignoramos los problemas filosóficos (Skinner, Sobre el conductismo 13-14).

${ }^{4}$ En 1916, Einstein escribió un obituario sobre Mach en el que lo elogiaba por haber empleado mucho tiempo estudiando tanto la ciencia en sí misma como la manera en que esta debería hacerse. Sin ese autoexamen, escribía Einstein... "Los conceptos que han demostrado ser útiles en el orden de las cosas, adquieren fácilmente autoridad sobre nosotros, de modo que olvidamos su origen terrenal contingente y los aceptamos como hechos inalterables. Tales errores a menudo bloquean el camino del progreso científico por largos períodos de tiempo" (Einstein, Ernst Mach 155).
} 
del conocimiento, y con el interés de avanzar en su empresa de unidad metodológica y de objeto de todas las ciencias.

De manera tal que no se justifica retomar de manera aislada y sesgada algunos de los principios epistemológicos de Mach. Le hubiera ayudado mucho a Skinner entender que dichos principios estuvieron muy influenciados tanto por una reflexión filosófica seria, como por el trabajo en psicología fisiológica, y que no hay manera posible de erigir una epistemología si no se tiene en cuenta la contribución integrada de la mente y de las experiencias sensoriales (dos aspectos que el conductismo se ufanó de haber desterrado del objeto de estudio de la psicología).

\section{Freud}

Las influencias de Mach en la obra de Freud se evidencian claramente en dos áreas, por un lado, en relación con la asimilación de algunos de sus principios epistemológicos y, por otro, en relación con su teoría energética.

Freud adoptó el modelo epistemológico de Mach, particularmente de los primeros capítulos de Conocimiento y error, uno de sus libros centrales sobre filosofía de la ciencia. Para Freud, Mach constituyó una referencia epistemológica más que un modelo a seguir en cuanto a su propuesta propiamente científica (Assoun 84-102). Freud retoma para justificar su propio proceder "científico" lo que Mach plantea como filósofo de la ciencia: defiende la idea de tomar como punto de partida unos principios meramente convencionales o arbitrarios, que son provisionales, en tanto podrán ser modificados a partir del curso de la experiencia.

Al principio deben comportar cierto grado de indeterminación; no puede pensarse en ceñir con claridad su contenido. Mientras se encuentran en ese estado, tenemos que ponernos de acuerdo acerca de su significado por la remisión repetida al material empírico del que parecen extraídas, pero que, en realidad, les es sometido (...) entonces quizá haya llegado la hora de acuñarlos en definiciones. Pero el progreso del conocimiento no tolera rigidez alguna, tampoco en las definiciones. Como lo enseña palmariamente el ejemplo de la física, también los «conceptos básicos» fijados en definiciones experimentan un constante cambio de contenido (Freud, Pulsiones 113).

Ahora bien, la tarea de modificar los conceptos básicos o principios teóricos a partir de lo que vaya sugiriendo en el curso de la experiencia 
sí fue seguido al pie de la letra por Mach en sus investigaciones físicas, y no tanto por Freud, como veremos en seguida.

Freud suscribe aquí el postulado de una cierta arbitrariedad como punto de partida en la ciencia y, asimismo, el principio de economía que plantea que solo se deben utilizar los principios estrictamente necesarios, de acuerdo con las necesidades que plantee la adecuación a la experiencia. En apariencia es metodológicamente claro pues manifiesta también su acuerdo con la idea según la cual se avanza en tanto se adapten los pensamientos a los pensamientos (como lo hizo en su metapsicología), pero también los pensamientos a los hechos, en tanto se los remita "de manera repetida al material empírico", como lo menciona en la cita. Sin embargo, es claro que, a pesar de que se suscribe teóricamente a este último ajuste, no lo llevó a la práctica en su investigación de la psique humana.

A diferencia de Skinner, Freud se aleja del rechazo machiano de la especulación teórica. El psicoanalista vienés insiste recurrentemente en la idea de una dominación de las ideas sobre el material empírico, puesto que aquellas no se sacan "únicamente de la experiencia actual". Si bien es cierto que en este punto Freud hizo una valiosa defensa de un punto de vista racionalista en la ciencia que, como ya anotamos, Einstein defiende y le reclama a Mach, su impulso lo llevó demasiado lejos hasta la construcción de un sistema teórico cerrado a las críticas y a la posibilidad de revisión y contrastación con base en casos nuevos o excepcionales. Como es sabido, este punto fue analizado in extenso por Popper (203-220), justamente para mostrar cómo la teoría de los sueños de Freud constituye un pleno ejemplo de verificacionismo que incluye entre sus problemas más importantes la falta de actitud crítica ante las propias hipótesis teóricas.

No obstante, Freud no dejaría de comparar al psicoanálisis como ciencia con la nueva física. "En nuestros días vivimos idéntica situación en la física cuyas intuiciones básicas sobre la materia, los centros de fuerzas, la atracción y conceptos parecidos están sujetos casi a tantos reparos como los correspondientes del psicoanálisis" (Freud, Narcisismo 75). Tal vez hubiese sido productivo para el psicoanálisis que Freud prestara atención a una línea crítica que no se dirigía tanto a atacar sus "intuiciones básicas", como a la manera un tanto dogmática en la que él se empeñó en defender sus elucubraciones teóricas, resistiéndose a las críticas y a la evidencia contundente en su contra. 
Por otro lado, tenemos el tema de las nociones de energía y fuerza psíquica. Como ya se había mencionado, las ideas de Mach no solamente promovieron grandes cambios en la investigación en física, sino que también fueron el sustento de la transformación de la concepción de la naturaleza que se tenía hacia finales del siglo XIX. Se trató de un giro hacia una concepción dinámica y no ya mecánica. Esto en contra del paradigma establecido desde la física newtoniana, en el cual la materia se definía en relación con los rasgos cuantitativos de la misma y el movimiento de partículas y fuerzas. En oposición a esto, Mach les dio prelación a los rasgos cualitativos, enfocándose en las sensaciones, originando con ello una nueva concepción de sujeto como una "corriente de sensaciones":

Una nueva forma de comprender la percepción, la sensación y la sensibilidad fue a través de la idea de que la electricidad y los nervios son eléctricos. Esto produjo significados de género particulares y contradictorios. Christoph Asendorf muestra que una idea central del modernismo vienés fue la nueva concepción del cuerpo como un conjunto de nervios y la realidad como simplemente un conjunto de estímulos. Esto se derivó del trabajo de Hermann Bahr y de The Analysis of Sensation (1885) de Ernst Mach en el que el ego era visto simplemente como una ficción; la idea de que el individuo está formado por corrientes de sensación reemplaza la idea del sujeto unificado (Henning 32).

Si tenemos en cuenta la influencia común que ejerció tanto en Mach como en Freud el trabajo de Brücke, quien sostenía una doctrina herbartiana de "fuerzas" psíquicas y energías mentales, comprenderemos por qué el enfoque freudiano sobre algunas enfermedades mentales, particularmente de las neurosis y de la histeria, se orientó hacia una explicación de las diferentes vías de expresión y destinos que alcanzan las energías psíquicas de estos pacientes y, en particular, de su energía sexual.

Para Freud todos los procesos mentales son de naturaleza energética. Todo proceso mental, llámese emoción, deseo, imaginación, formación de ideas, etc., debe ser considerado como una modificación de energía. Tiene una carga de energía mental y, dado que su pretensión era tomar el modelo científico de explicación de los procesos físicos y aplicarlo a los procesos mentales, estos deberían estar gobernados por las mismas leyes que los procesos físicos ${ }^{5}$. Pero, como es sabido, a pesar de que

${ }^{5}$ Cfr. Wilkes (1975), quien plantea que Freud nunca abandonó su fe en la verdad del fisicalismo. También Cavell (1993) y Assoun (2001) han mostrado la persistencia de Freud en encontrar el correlato neurofisiológico de los conceptos psicoanalíticos. 
Freud tuvo un fuerte interés inicial en hallar la contraparte física de la dinámica de la psique (así como de algunas instancias de su aparato psíquico: el yo, el ello y el superyó), finalmente resignó sus aspiraciones fisicalistas (Cely 136-144) y propuso entender una noción plena e independiente de 'energía psíquica'. La defensa de esta tesis por parte de Freud constituyó una de las motivaciones que llevaron a Breuer a separarse de Freud: “Breuer, así como Mach, era un psicofísico opuesto a la nueva psicología profunda y a lo que presuponía: un cambio de interés psicológico de la sensación, -tan desconcertante para du Bois-Reymond-, a la representación y la necesidad de un signo y símbolo hermenéutico" (Cappelletti 220). Fue de esta manera que se produjo en Freud el desplazamiento de todo su interés hacia el poder curador de la palabra y hacia una explicación del aparato psíquico en términos de representaciones conscientes e inconscientes, y del flujo de energías, pero psíquicas. El psicoanálisis terminó así reduciendo a representaciones todo lo relacionado con las sensaciones y el cuerpo, algo que tuvo repercusiones importantes en términos de la teorización de lo psicológico y de la aplicación clínica de esos conocimientos ${ }^{6}$.

Tenemos hasta ahora un empirismo reacio a las teorías con Skinner y un "racionalismo" renuente a la confrontación con la experiencia con Freud. Al final del texto veremos cómo esta desacertada interpretación de los principios machianos por parte de estos dos autores tuvo la desafortunada consecuencia, para la psicología, de desviar la atención que se merecía la tesis tan central en Mach del estudio de las sensaciones desde un punto de vista empírico y experiencial. En seguida veremos cómo se pueden articular algunos principios de epistemológicos de Mach con la fenomenología, y algunas tesis centrales de la psicología de la Gestalt.

\section{Fenomenología y Psicología de la Gestalt}

Es importante entender que la epistemología y la propia investigación científica de Mach, tanto en física como en psicología, tuvieron más que ver con un trasfondo de comprensión de la realidad que podemos llamar fenomenalista o fenomenológico y no con uno realista ingenuo (tal como en otros positivismos). De hecho, es clara la mutua influencia entre las

\footnotetext{
${ }^{6}$ Cfr. Fuchs (2011) para una crítica importante que apunta a mostrar cómo en el psicoanálisis freudiano se desplazó la importancia de las pulsiones como energías corporizadas a una consideración de las mismas meramente como representaciones que están ocultas en un lugar de la psique, entendida como un 'aparato interno'.
} 
concepciones de Mach y la fenomenología husserliana. De un lado, Husserl reconoció que el análisis de las sensaciones de Mach preparó el camino para el método fenomenológico ${ }^{7}$. Y, de otro, puede caracterizarse el punto de partida de Mach como fenomenológico al orientarse hacia el entendimiento de los fenómenos en su realidad psicofísica, tal cual son experienciados. En cualquier, caso, se ha considerado más apropiado su denominación como fenomenalista y no como fenomenólogo dado que, si bien es cierto se concentró en los fenómenos, no hizo eco de las aspiraciones trascendentales de la fenomenología husserliana y se lo debe considerar más bien como un fenomenalista científico.

Ambas fenomenologías, las de Mach y Husserl consideraban el campo fenoménico como el punto de partida de las consideraciones filosóficas y rechazaban el concepto de "cosas en sí mismas". Además, ambos adoptaron el método de descripción pura sin presupuestos metafísicos. Pero la fenomenología de Mach carecía por completo del momento trascendental en comparación con la de Husserl. Al contrario de él, Husserl demandó un cambio de actitud, de la "actitud natural" a la “actitud trascendental" (Noé, Mach's Relativism 237-238).

Existió una coincidencia, de todas maneras, entre Mach y la fenomenología respecto a los orígenes experienciales del conocimiento. Para Mach, la materia se definía como reacción o interrelación de las percepciones sensoriales: "La ciencia del calor, argumenta, se deriva del concepto de sensación de calor, la ciencia de la luz a partir del concepto de la intensificación de la iluminación, la ciencia de la acústica desde el concepto de frecuencia, y así sucesivamente" (Mulligan \& Smith 135). Para Mach, la sensación revela el mundo, un mundo que, entendido así, no se puede deducir.

Ahora bien, una vez dilucidado el origen experiencial del conocimiento, los caminos de Mach y Husserl se separan. Como ya anotamos, Husserl se orientó por la búsqueda de una fundamentación trascendental de las estructuras de la experiencia, mientras que Mach continuó más bien con la intención de indagar empírica y experimentalmente ese

\footnotetext{
7 "Husserl estaba familiarizado con el uso de Mach del término «fenomenología», incluso al principio de su carrera; pero más adelante, en sus lecciones de Ámsterdam de 1929, explícitamente reconoció a Mach como el precursor de la fenomenología" (Moran 7). Hay que señalar, no obstante, que así como Husserl estuvo presto a reconocer una primera influencia de Mach y el valor de sus aportes en labores histórico-epistemológicas, también fue tajante en su crítica respecto a su principio de economía en la ciencia (cf. Husserl, Investigaciones lógicas §55).
} 
amplio campo de las sensaciones. Esta línea de trabajo atrajo el interés de psicólogos como Piaget y Ehrenfels quienes consideraron de mayor importancia el análisis de la percepción realizado por Mach (Ehrenfels 121). En el caso de la psicología de la Gestalt, en particular, la idea de la percepción como relacional será retomada como fundamento de su tesis central según la cual percibimos formas como un todo (cf. Mulligan \& Smith 128). En Análisis de las sensaciones (1984) Mach plantea esta idea de forma clara: entre nuestra estructura cognitiva y la experiencia existe una relación dinámica; la mente y los sentidos contribuyen activamente en la sensación. Con el experimento de las 'bandas de Mach' nuestro autor demostró que las ilusiones ópticas no se explicaban por errores en el juicio o procesos cerebrales, sino que se trataba de errores de los sentidos: "El significado inmediato de esto fue que proporcionó un fuerte argumento en contra de las teorías representacionales directas de la percepción. Simplemente no hay isomorfismo entre la realidad y la apariencia". (Pojman, Ernst Mach 7).

No es difícil ver acá la conexión con el principio central de la psicología de la Gestalt: percibimos siempre de manera inmediata formas, totalidades, esto es, unidades de sentido ya estructuradas, no elementos aislados que después unificamos por la actividad de la conciencia, según lo plantean varias líneas de asociacionismo. Con ello la psicología de la Gestalt supera la hipótesis de constancia y reconoce la contribución activa del sujeto en la construcción de esas estructuras totales que permiten la percepción con sentido.

La puntada que nos permite aquí conectar tesis centrales de Mach con la psicología de la Gestalt y con la fenomenología de Husserl y Merleau-Ponty es la que proviene del hilo de una crítica común a una epistemología y una ontología inscritas en el marco de los dualismos mente/objeto y mente/cuerpo, así como del rechazo de una concepción materialista mecanicista ${ }^{8}$. Desde la época moderna estos tipos de dualismo habían hecho carrera, estipulando que las sensaciones correspondían al dominio de lo subjetivo como cualidades secundarias y que el científico “... podría ignorar la cualidad de la experiencia como tal y concentrarse en modelos matemáticos de las cualidades primarias de la naturaleza: masa, número, forma y movimiento" (Banks IX). El análisis de la percepción como un fenómeno relacional contribuyó

\footnotetext{
${ }^{8}$ Dos concepciones a las cuales se opuso fervientemente Merleau-Ponty en su Fenomenología de la percepción (1985).
} 
así a superar, de un lado, los problemas asociados al racionalismo y su imposición de condiciones a priori y, de otro, los relacionados con un empirismo asociacionista que defendía una explicación de la percepción como agregado de partes en un sujeto pasivo.

Mach llevó a cabo un análisis de las sensaciones como elementos constitutivos de la realidad psicofísica, pero entendiendo que estos elementos configuran complejos cuya formación es una función del sistema como un todo. En el dibujo de la construcción en perspectiva de lo que él mismo ve con su ojo izquierdo (incluido en su Análisis de las sensaciones, figura 1) se aprecia a un Mach interesado en captar la totalidad del espacio perceptivo que emerge en el encuentro entre un perceptor con sus cualidades particulares (en este caso él mismo percibiendo solo con su ojo izquierdo después de la apoplejía que sufrió y que le dejó paralizado medio cuerpo) y lo percibido - en poner "la visión en lo visible", como diría Merleau-Ponty (1986).

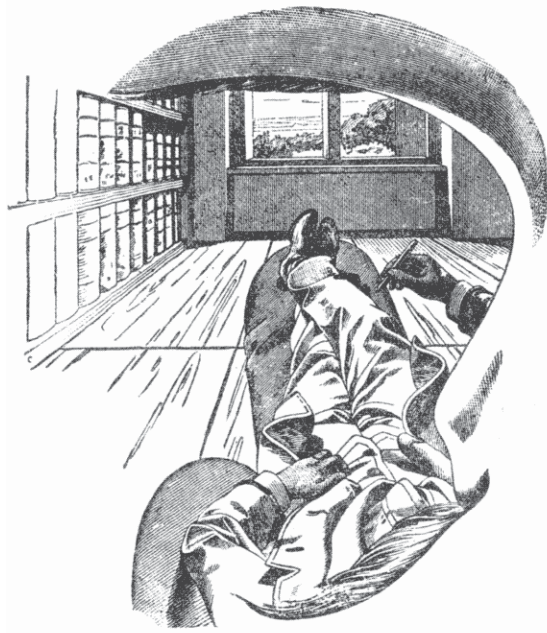

Fig. 1. Dibujo realizado por Mach en el que representa su espacio perceptivo tal como es captado por su ojo izquierdo, incluido en su libro The Analysis of Sensations and the Relation of the Physical to the Psychical de 1886.

De ahí que sus esfuerzos estuvieron dirigidos a encontrar la unidad de las ciencias en torno a la tarea, común a la física y a la psicología, de establecer a las sensaciones como los elementos últimos constitutivos de la realidad. La fenomenología, por su parte, se ocupará de realizar una descripción de la experiencia corporizada vivida, con sus rasgos particulares dinámicos y afectivos (Merleau-Ponty 1985). Esta vía de 
aprehensión de la experiencia -que incluso se le escapa en toda su riqueza al método fenomenológico mismo- es difícil de conciliar con las pretensiones científicas al estilo de las machianas. De ahí que en MerleauPonty sean constantes las analogías de la actitud fenomenológica más bien con las potencialidades expresivas del arte: cada una, a su manera, constituye una forma de aprehensión del mundo, de los otros y de la propia subjetividad corporizada en toda su riqueza experiencial. Es por ello que Merleau-Ponty afirmará que la ciencia tiene mucho que aprender de esta captación diferencial de la experiencia a partir de la descripción fenomenológica y del arte (Merleau-Ponty El ojo y el espíritu 19).

Pues bien, lo que me gustaría sugerir aquí para terminar es que el horizonte de posibilidades futuras de la psicología puede aparecer como prometedor si se llega a hacer realidad el proyecto de Husserl de una fenomenología que contribuya a que la psicología se convierta en una ciencia bien fundada y auténtica, con la participación de enfoque científico de las sensaciones y de la visión del arte.

\section{Conclusiones}

Cuando la filosofía de la ciencia proveniente de diferentes líneas coincidió en rechazar la metafísica, dirigió su atención a lo que hoy en día se llama una epistemología naturalizada. Puesto que se consideró que ya no era útil plantear esencias ideales o fundamentos trascendentales para entender las bases del conocimiento, se orientó hacia la tarea de comprensión de los fundamentos del conocimiento en el sujeto epistémico real, ese que tiene experiencias vividas del mundo y que integra sensaciones y pensamientos en las tareas de conocimiento. Para tal fin se recurrió hacia finales del siglo XIX y comienzos del XX a los aportes de científicos como Fechner y, por supuesto, a los del propio Mach quien, bajo la influencia de aquel, estudió la relación de nuestras sensaciones con los estímulos externos.

En este sentido, se hubiera podido reseñar en la historia de la psicología de las primeras décadas del siglo XX un momento clave, iniciación de su progreso como ciencia y de su ascenso hacia la cumbre de la buena fama: la psicología como ciencia base, fundamento de todas las demás ciencias -y también de la matemática y de la lógica9 ${ }^{-}$. Y esto teniendo en cuenta que, como lo vimos, la filosofía de la ciencia de Mach estuvo

\footnotetext{
${ }^{9}$ Como lo pretendía Piaget en su exposición del 'círculo de las ciencias' (cf. su Introducción a la epistemología genética).
} 
mucho más motivada por las investigaciones en psicología experimental de la época (del estilo de la psicofísica Fechner) que por las de la física. Pero no sucedió así. El recorrido que propusimos aquí nos permitió aportar una razón para explicar por qué hoy en día contamos una historia diferente de la psicología: de un lado, tomó el camino supuestamente científico del conductismo, con su empirismo recalcitrante, surgido de una pobre interpretación de la filosofía de la ciencia de Mach y ciego, por ello, a la estructura total del comportamiento. Y, de otro, con el psicoanálisis freudiano tomó la opción de construirse como una teoría tercamente auto verificada que, además, terminó reduciendo toda conducta y toda experiencia corporizada a la palabra. En síntesis, contamos hoy en día la historia de una psicología "científica" que con el conductismo redujo su objeto de estudio a conductas observables, y con el psicoanálisis y el cognitivismo temprano a los procesos mentales, cognitivos o representacionales de un sujeto psicológico descorporizado. Aún no es tarde para contar una historia diferente de una nueva psicología, fundada fenomenológicamente y estimulada por las investigaciones llevadas a cabo desde los enfoques corporizados y enactivos y enriquecida por las artes.

\section{REFERENCIAS}

Assoun, Paul Laurent. Introducción a la epistemología freudiana. Ciudad de México: Siglo XXI, 2001.

Blackmore, John. (Ed.). Ernst Mach. A Deeper look. Documents and New Perspectives. Dordrecht: Kluwer, 1992.

Cappelletti Vincenzo. “Discovery and Vocation”. On Scientific Discovery. The Erice Lectures 1977. M. Grmek et al., editores. Dordrecht: D. Reidel, 1977. 211-226.

Cavell Marcia. La mente psicoanalítica. De Freud a la filosofía. Ciudad de México: Paidós, 2000.

Cekic Miodrag. "Mach's Phenomenalism as a link between Physics and Psychology". Ernst Mach. A Deeper look. Documents and New Perspectives. J. Blackmore, editor. Dordrecht: Kluwer, 1992. 201-214.

Cely, Flor Emilce. "Razones y causas en el psicoanálisis freudiano". Eidos. Revista de Filosofía de la Universidad del Norte. 23, 2015: 137-158.

Chomsky Noam. "Crítica de «Verbal Behaviour» de B. F. Skinner". ¿Chomsky o Skinner? La génesis del lenguaje. Barcelona: Fontanella, 1977. 
Ehrenfels Christian von. "On 'Gestalt Qualities'” ('Ober Gestaitqualitiiten', originalmente publicado en Vierteljahreschrift for wissenschaftliche Philosophie 14 (1890), 242-292). Traducción recuperada del sitio https://pdfs.semanticscholar.org/178b/ b13c2f299c5a544d1c5339cff9eaae2be13c.pdf

Einstein Albert. "Ernst Mach". Ernst Mach. A Deeper look. Documents and New Perspectives. J. Blackmore, editor. Dordrecht: Kluwer, 1992. 154-159 (original de 1916).

Freud Sigmund. Introducción al narcisismo. Buenos Aires: Amorrortu, 1976a.

Freud Sigmund. Pulsiones y destinos de la pulsión. Buenos Aires: Amorrortu, 1976b.

Fuchs Thomas. "Body Memory and the Unconscious." Founding Psychoanalysis. Phenomenological Theory of Subjectivity and the Psychoanalytical Experience. D. Lohmar y J. Brudzinska, editores. Dordrecht: Kluwer, 2011. 69-82.

Henning Michelle. “Don't Touch Me (I'm Electric): On Gender and Sensation in Modernity". Women's Bodies Discipline and Transgression. Jane Arthurs \& Jean Grimshaw, editoras. London: Cassell, 1999. 17-47.

Husserl Edmund. Investigaciones lógicas. Madrid: Alianza, 1982.

Mach Ernst. "A Draft Foreword to the Russian Translation of Die Analyse der Empfindungen". Ernst Mach. A Deeper look. Documents and New Perspectives. J. Blackmore, editor. Dordrecht: Kluwer, 1992. 115-117 (original de 1906/1907).

Mach Ernst. "Sensory Elements and Scientific Concepts". Ernst Mach. A Deeper look. Documents and New Perspectives. J. Blackmore, editor. Dordrecht: Kluwer, 1992. 118-126 (original de 1910a).

Mach Ernst. "The Leading Thoughts of my Scientific Epistemology and its Acceptance by Contemporaries". Ernst Mach. A Deeper look. Documents and New Perspectives. . J. Blackmore, editor. Dordrecht: Kluwer, 1992. 133-139 (original de 1910b).

Mach Ernst. Conocimiento y error. Buenos Aires: Espasa-Calpe, 1948.

Mach Ernst. The Analysis of Sensations and the Relation of the Physical to the Psychical. LaSalle, IL: Open Court, 1984.

Merleau-Ponty Maurice. El ojo y el espíritu. Madrid: Trotta, 2013. 
Flor Emilce Cely Á.

Merleau-Ponty Maurice. Fenomenología de la percepción. Barcelona: Planeta-Agostini, 1985.

Merleau-Ponty Maurice. La estructura del comportamiento. Buenos Aires: Hachette, 1953.

Moran Dermot. Introducción a la fenomenología. Barcelona: Anthropos, 2011. Mulligan Kevin. \& Barry Smith. "Mach and Ehrenfels: The Foundations of Gestalt Theory". Foundations of Gestalt Theory. B. Smith, editor. Munich: Philosophia, 1988.

Noé Keiichi. "Mach's Relativism vs. Apriorism and the Mechanistic World View". Ernst Mach. A Deeper look. Documents and New Perspectives. J. Blackmore, editor. Dordrecht: Kluwer, 1992. 229-241.

Piaget Jean. Introducción a la epistemología genética. Buenos Aires: Paidós, 1978.

Pojman Paul. "Ernst Mach". Stanford Encyclopedia of Philosophy. Web 18 jun. 2017 <https:/ / plato.stanford.edu/entries/ernst-mach/> 2009.

Popper Karl. Post Scriptum a la lógica de la investigación científica. Realismo y el objetivo de la ciencia. Buenos Aires: Paidós, 1985.

Skinner Burrhus Frederic. Science and Human Behavior. New York: Macmillan, 1953.

Skinner Burrhus Frederic. Beyond Freedom and Dignity. London: Penguin Books, 1976.

Skinner Burrhus Frederic. Sobre el conductismo. Barcelona: Planeta, 1987. Skinner Burrhus Frederic. Tecnología de la enseñanza. Barcelona: Labor, 1970. Smith Laurence. Conductismo y positivismo lógico. Bilbao: Desclée de Brouwer, 1994.

Wilkes Kathleen. "Anthropomorphism and Analogy in Psychology". The Philosophical Quarterly. 25 (99), 1975: 126-137.

\section{Como citar:}

Cely Flor Emilce "La influencia del empiriocriticismo de E. March en la fundación de la psicología". Discusiones Filosóficas. Jul.- Dic. 20 (35), 2019: 91-110. DOI: 10.17151/difil.2019.20.35.6. 